\title{
Planar Reactive Magnetron Sputtering to Obtain Dielectrics and Transparent Conductive Thin Films
}

\author{
Marina Kipiani
}

\begin{abstract}
The design and technological capabilities of the innovative planar magnetron sputtering device (PMSD) with a rotating magnetic unit are considered. Namely, technological capabilities for obtaining modern materials, such as dielectric and transparent conductive thin films. The article describes innovative approaches to solving such problems that are associated with reactive sputtering, and it is difficult to solve them under the conditions of stationary magnetron sputtering. The main advantages of the rotating magnetron device developed by us are: The compactness of the device, the effective cooling of the spray target, and the cathode assembly as a whole, as well as the use of a cooling system to set the magnetic unit in motion. The rotation of the magnetic system under the atomized round target cathode provides synchronous movement of a closed magnetic field along its surface; By adjusting the distance between the permanent magnets of the opposite poles and the configuration of their location, you can change the configuration of the magnetic field on the target surface. This allows you to adjust the distribution of the intensity of ion bombardment on the surface of the target. In turn, this, together with the regulation of the rotation speed of the magnetic system, opens up new possibilities for controlling the technological regimes of reactive sputtering; provides flatness of the bottom of the sprayed target and high uniformity in the composition of thin dielectric and transparent conductive films. These design features can significantly improve the technical characteristics of a planar magnetron and apply it to solve a wide range of reactive sputtering problems from applying high-quality, required coatings to obtaining uniform high-quality dielectric and transparent conductive thin films.
\end{abstract}

Index Terms - Magnetron, reactive, sputtering, dielectrics, target, films.

\section{A BRIEF History OF THE MAGNETRON IN THE WORLD}

The history of thin films, their production and use dates back several thousand years. In ancient Egyptian tombs, copper and bronze statues, jewelry and religious artifacts, covered with gold films dating from the middle of the Bronze Age, were found.

The functional characteristics of thin films directly depend on the synthesis methods used and the conditions for their deposition. At the same time, obtaining thin-film structures with desired characteristics requires systematic research in the field of synthesis and the study of their physical and physicochemical properties.

The interest and need for the practical use of thin films are due to the presence of such unique characteristics that cannot be achieved in bulk materials from which various structures

Marina Kipiani, Senior Researcher, Deputy Head of Optical Research, LEPL Scientifica Research Institute, Tblisi, Georgia are made. Moreover, without thin films it is impossible to imagine the possibilities of many modern materials.

In the seventies of the last century, a flat magnetron was described in a patent by John Chapin and later he presented it in an industrial journal in his article. The device was a rectangular magnetron with water cooling with a closed field, which led to a continuous rectangular erosion profile on the surface of the target. This work can be considered a step forward in the production of the magnetron, although it had many shortcomings.

Our proposed method can be very successfully used to solve problems arising in the process of reactive spraying, which for many years, scientists and technologists have tried to solve.

The works of Berg and his colleagues from Uppsala University are interesting, in which it was found that the dynamics of reactive sputtering does not coincide with the dynamics of film growth. It is noteworthy that metalloid anionic particles behave in such a way that thick metalloid layers can accumulate on the target without strict regulation of the processes, which leads to the formation of films having a stoichiometric, almost pure metalloid in their composition. In addition, the presence of hydrogen can lead to reverse reactions both on the target surface and on the surface of the growing film, which impedes the development of processes. Based on this, during jet spraying, among other factors, it is very important to be very careful when regulating the partial pressure of a reactive gas.

In the last years, vacuum deposition has become the main method of creating thin films for use in science, R\&D (Research and Development), and industry. This method gives ample opportunities, it allows you freely change the conditions in which the deposition occurs, change the sputtered materials, etc. This direction includes the basics of modern vacuum microelectronics technologies - deposition in vacuum and vacuum plasma-chemical treatment of thin-film structures. In this work, we will try to present PMSD technology as a way to solve the problems facing reactive sputtering, which is important to obtain dielectrics and transparent conductive thin films.

\section{A BRIEF HiSTORY OF THE MAGNETRON IN GEORGIA}

Development of thin-film coating technology in our country is connected with our centuries-old culture, science and the development of modern microelectronics. As well as important international experience.

It is important that in the repositories of our national museum we meet unique archaeological and ethnographic 
exhibits, analyzing which we understand that since ancient times there was an interest in objects decorated with precious metals or made of precious metals (Fig. $1 \mathrm{a}, \mathrm{b}$ ). This fact once again emphasizes our cultural traditions and place in ancient civilization.

I demonstrate a sample (Fig. 1c) in the form of ceramics coated with a thin layer (several microns) of silver, preserved

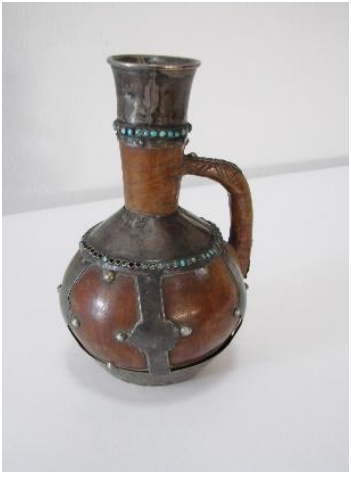

a)

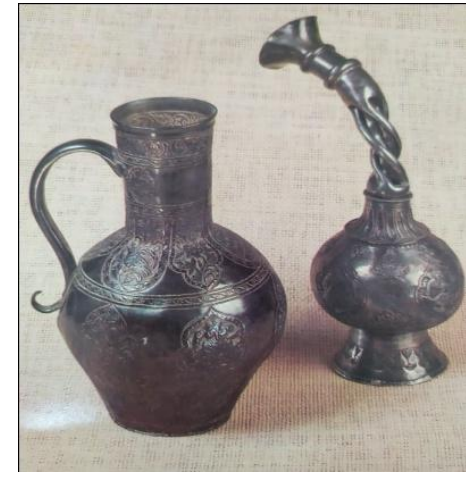

b) from the 90s of the last century, which was obtained using this modern magnetron sputtering technology, discussed in this article. Using this technology, you can get the same visual effect at the lowest cost, because the minimum amount of silver is spent.

Fig.1. Ancient ethnological samples of the State Museum of Georgia:

a) decorated with silver; b) silver.

c) Modern the thinnest silver-plated ceramic produced using our innovative technology.

What is unique in the assessment of ethnologists and archaeologists, in museum repositories, such an exhibit covered with a thin layer of expensive metal does not exist.

It should be emphasized that another technology for thin-film coatings of household items, in terms of quality and ecology, does not exist.

Based on the foregoing, we can say that the scientific success in the technology for the production of thin films in our country is not surprising and not accidental.

It is also important to take into account international experience, which played a large role in this area of engineering, technology and modern production in Georgia.

It is interesting that the development of magnetron sputtering in the modern history of our country is also associated with the development of microelectronics in recent years.

The development of magnetron sputtering in the second half of the last century to the present time is mainly associated with the transition to planar magnetron sputtering systems. As is known, magnetron sputtering allows the use of cathodes made of metals, as well as alloys and various compounds, and reduces the substrate temperature by $200 \mathrm{~K}$. It is important that the degree of ionization, the kinetic energy of ions, and the deposition rate during magnetron sputtering using conventional magnetrons are lower than when using plasma with an arc discharge.

In the 80s of the last century, georgian scientists developed the original design and prototype of a planar magnetron spraying device (PMSD) with a rotating magnetic unit. In a modernized, innovative device, the coolant flow is used to rotate a whole magnetic block [1, 2].

This device continues to evolve to the present day and has reached such levels from which it is possible to solve many unresolved problems associated with the production of modern vacuum technology.

\section{Planar Magnetron SputTering DeVICE}

The modern world cannot be imagined without thin-film coatings. They are used to improve the physical and chemical properties of materials, in microelectronics, optics, in the form of transparent and conductive oxide and antireflection layers in solar cells, as well as in many other fields of science and technology. The use of thin-film materials is an effective way to solve the problem of miniaturization, to obtain various important devices for different purposes and to reduce the consumption of materials by devices for various purposes. The interest in the practical use of thin films is due to the presence of a number of unique characteristics that are absent in the entire volume of materials from which such structures are made. The physical properties and functional characteristics of thin films are determined by their conditions and the methods for their preparation. Scientists already agree that the formation of thin-film structures with specified characteristics requires systematic research in the development of magnetron devices and various technological methods of synthesis, as well as the study of the physical and physicochemical properties of the necessary materials.

In fact, the development of magnetron sputtering from the second half of the last century to the present time is associated with the development of flat magnetron sputtering systems. In the 70s, first J. F. Korbani [1], and then J. S. Chapin [4,5] proposed the design of a magnetron sputtering device. The principle of operation of the structure is shown in Fig. 2. 


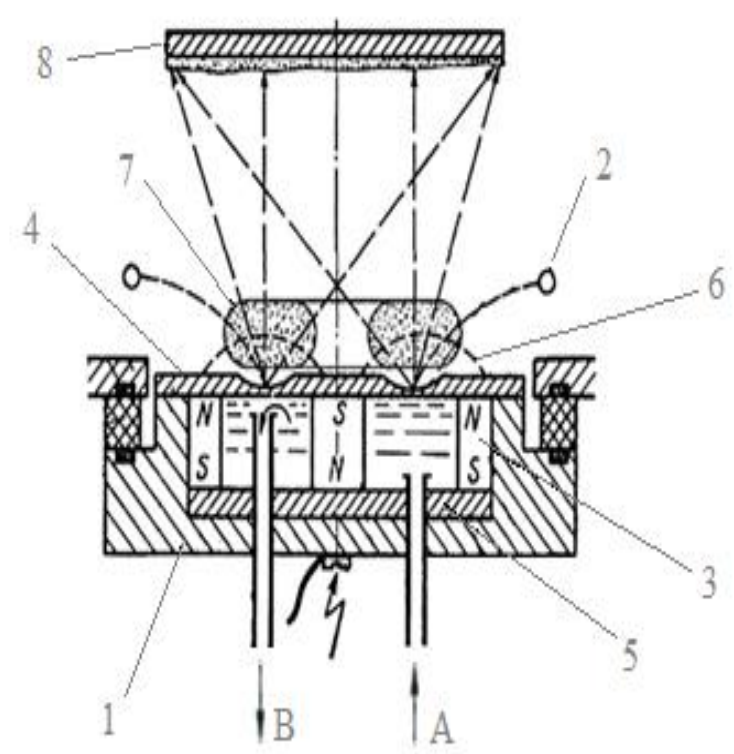

Fig.2. Flat-cathode magnetron spraying apparatus.

1 - cathode assembly; 2 - anode; 3 - permanent magnets; 4 - target; 5 - holder of the magnetic system; 6 - configuration of the magnetic field; 7 - magnetron plasma; 8-substrate; A coolant inlet; B - coolant outlet.

The stability and magnitude of the magnetron discharge and the reproducibility of the process of applying thin films depend on the magnitude and stability of the parameters in similar technological processes. These parameters are always interconnected. The voltage at the electrodes, the discharge current, the current density at the target, the specific power, the magnitude of the magnetic field induction and the pressure of the working gas are the main characteristics of a planar magnetron spray device. For a flat cathode system, the current density at the target is very high and amounts to about $200 \mathrm{~mA} / \mathrm{cm}^{2}$. Given the heterogeneity and the fact that the maximum current density in the central part of the sputtering zone can be much higher, as a result, this leads to thermal overload of the curved target. In places of poor contact with the holder leads to its melting.

One of the main elements of the device (along with the cathode - the target and the anode) is a magnetic system that forms a field of a given configuration and magnitude on the surface of a circular target to create an effective magnetic trap for electrons, i.e. the so-called "electronic treadmill."

The magnetic field configuration of the magnetic system of modern flat magnetron sputtering devices has a simple round or rectangular shape. After spraying on the target, a deep and narrow depression forms in the ring or rectangle. This fact shortens the life of the target, so the use of the target material is about $30 \%$ and significant damage. The erosion groove leads to an increase in the electric field strength on the target surface and to the effect of a hollow cathode for secondary electrons that oscillate between opposite walls of the groove. As a result of these processes, the voltage and discharge power, the energy of the ions bombarding the target, the sputtering rate of the target, and, naturally, the deposition rate of the coatings are reduced.

In the 80 s of the last century, the development of new designs of a magnetron spray device and coating technology, a flat magnetron spray device (PMSD) with a rotating magnetic block can be considered a significant success for Georgian scientists and technologists [4]. Original design developed, laboratory and industrial designs created. A good design feature is that the coolant flow, in addition to the main cooling function, is used in the cathode assembly to rotate the magnetic unit.

The presented innovative design of the PMSD with a rotating magnetic block (Fig. 3.) contains: an anode (5), a cathode assembly (1) with a sprayed round target in the cavity of the cathode assembly, a rotating magnetic assembly (using a coolant flow), a cooling block with a drain hole in the central part of the device, a flange with a hole and a holder of the magnetic system (2) in the form of a disk with blades are fixed on the cathode assembly in the lower part. In a row on both sides of the closed axial line there is a complex of permanent magnets (3), facing the magnetic drive with opposite poles.
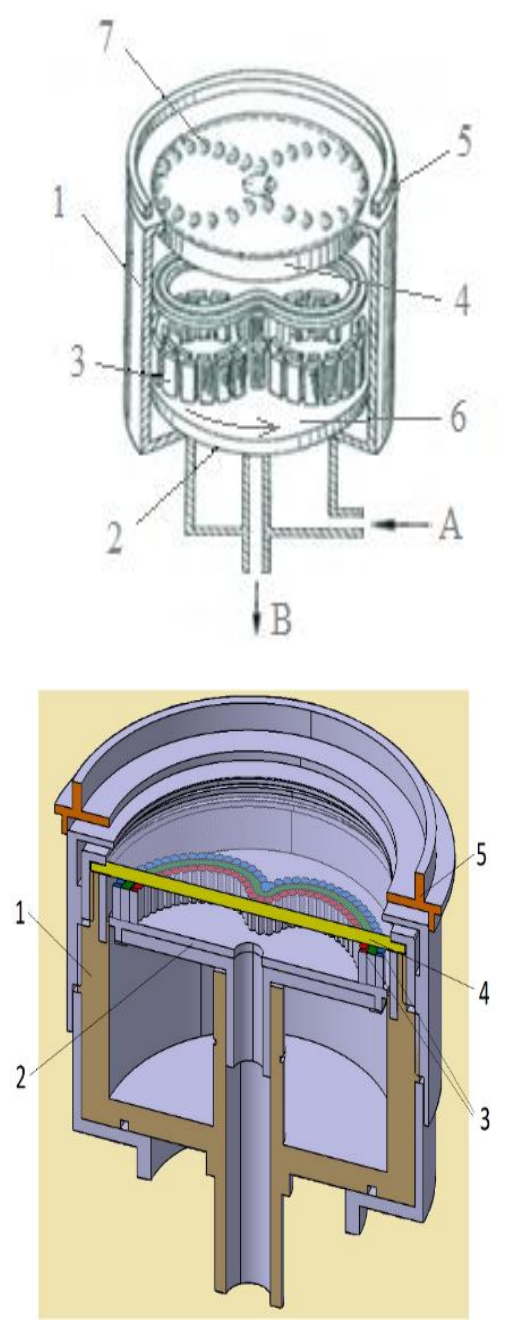

Fig. 3. Construction of PMSD with rotating magnetic block.

1 - cathode assembly, 2 - magnetic system holder, 3 - array of permanent magnets, 4 - annular target cathode, 5 - anode, 6 - magnetic conductor, 7 - plasma (area of erosion), A coolant inlet, B - coolant outlet.

This turbulent flow of coolant rises up in a spiral to the holder of the round target 4, washes it and the walls of the cathode assembly, then goes to the drain hole (arrow B). Possessing 
large-scale turbulence, the cooling liquid acquires an increased ability to absorb heat (approximately 3-4 times), and provides effective cooling of the sputtered target and the cathode assembly as a whole.

The rotation of the holder of the magnetic system under the target together with it leads to the synchronous movement of a closed magnetic field, that is, the movement of the plasma (7) on its surface. The configuration of the arrangement of the magnets was mathematically calculated, which led to the creation of a closed magnetic field, the intensity vector of which is parallel to the target surface in almost the entire region above the target. The surface of the target, located between the strips of the magnetic field, is intensively sprayed, has the form of a closed path, a closed erosion zone of a circular target in the form of a "treadmill for electrons." This provides a uniform erosion zone and the maximum possible target area [6].

When a constant voltage is applied between the target and the anode and an inhomogeneous electric field occurs, an abnormal glow discharge is excited. A closed magnetic field above the sprayed surface of the target allows you to collect the discharge plasma directly on the target. Under the influence of ion bombardment of the cathode, electrons appear that are instantly captured by a magnetic field. They move in a complex configuration on the target's surface along the treadmill until several ionizing collisions occur.

The volume of the vacuum chamber is pumped out to a pressure of 10-4-10-5 $\mathrm{Pa}$. Coolant supplied to the cathode assembly provides rotation of the magnetic unit, the speed of which can be adjusted from zero to $80 \mathrm{rpm}$. An inert gas, $\operatorname{argon}(\mathrm{Ar})$, is introduced into the vacuum chamber, and in the case of reactive magnetron sputtering, chemically active components are added, such as oxygen $\left(\mathrm{O}_{2}\right)$, nitrogen $\left(\mathrm{N}_{2}\right)$, carbon $\left(\mathrm{C}_{2}\right)$, etc. The generated working pressure in the vacuum chamber is ( 0.27-1.30) $\mathrm{Pa}$. When a constant (or pulsed) voltage is applied to the target cathode, a plasma appears, the configuration of which repeats the configuration of a closed magnetic field ("electronic treadmill") on the cathode and moves synchronously with it. During the process, the erosion zone covers all new, not yet sprayed areas of the target. Subsequently, more and more new areas are being sprayed intensively. The erosion area increases, the heat load per unit area decreases, the probability of curvature of the target decreases [6].

\section{Reactive Planar Magnetron Sputtering Device}

Reactive magnetron sputtering is currently a widely used and interesting process for the manufacture of mono- and multicomponent thin-film coatings. In the case of reactive spraying, often there is a need for intensive deposition at the lowest substrate storage temperature. To meet these requirements, it is very important to have flexible control and controllability of the deposition processes. The processes of reactive magnetron sputtering are not as simple and understandable as the processes of magnetron sputtering in an atmosphere of inert argon gas only. The addition of a reactive gas during jet spraying significantly changes the physical and chemical processes that occur on the surface of the target. The deposition rate and composition of the resulting coating are highly dependent on the flow of reactive gas. Too low a supply of reactive gas leads to intense atomization of the metal, but can lead to non-stoichiometric composition of the deposited film. While too high a supply of reactive gas allows one to obtain a stoichiometric composition of the deposited film, it leads to poisoning of the target surface and significantly reduces the deposition rate.

Most of the existing technological processes based on stationary flat magnetron sputtering encounter a hysteretic behavior of the deposition process during reactive sputtering. This is due to the interaction of the surface of a metal target with a chemically active gas during the sputtering process. This determines the hysteretic dependence of the partial pressure of the reactive gas on the voltage on the target and the deposition rate.

At a high deposition rate, to obtain stoichiometric films, it is necessary to control the spraying power and also by carefully controlling the flow of reactive gas in the chamber, which is very difficult and often not available [7].

A serious disadvantage of reactive magnetron sputtering is also the phenomenon of the so-called "sparking" since it can drastically limit the stability of the process.

Strong sparking can damage the surface of the target, as it can even lead to local melting of the metal material, and also cause point damage to the thin film. In addition to the formation of an arc, deposition by reactive spraying of insulating materials in direct current mode is accompanied by another significant problem, which is often described by the term "disappearing anode".

In the magnetron sputtering system, an existing electrode (anode) collects electrons from the discharge, and as a result, the electron flow closes the electric circuit from the power source. In the immediate vicinity of the cathode, for example, a screen, chamber walls, etc., serve as this electrode.

In stationary reactive magnetron deposition, all internal surfaces may eventually become coated with an insulator, and the anode will disappear. In this case, the discharge resistance will increase, the distribution of the plasma glow will become uneven, the potentials on the electrodes and other process parameters will become unstable, and all these processes will affect the properties and qualities of the deposited films.

Berg and his colleagues [8] simulated those serious disadvantages of reactive magnetron sputtering, which include gettering and chemisorption of a reactive gas on the surface of a metal target..

In reactive ion implantation, when chemisorption of a reactive gas on the surface of a metal target is observed at a high deposition rate (low current and high voltage), the sputtering rate of the target decreases and its poisoning increases. Whereas at a low deposition rate, gettering dominates, and the effect of chemisorption on the targets is less pronounced. Figure 4 shows how energetic ions (with several hundred eV) penetrate the target and cause a cascade of collisions there. 


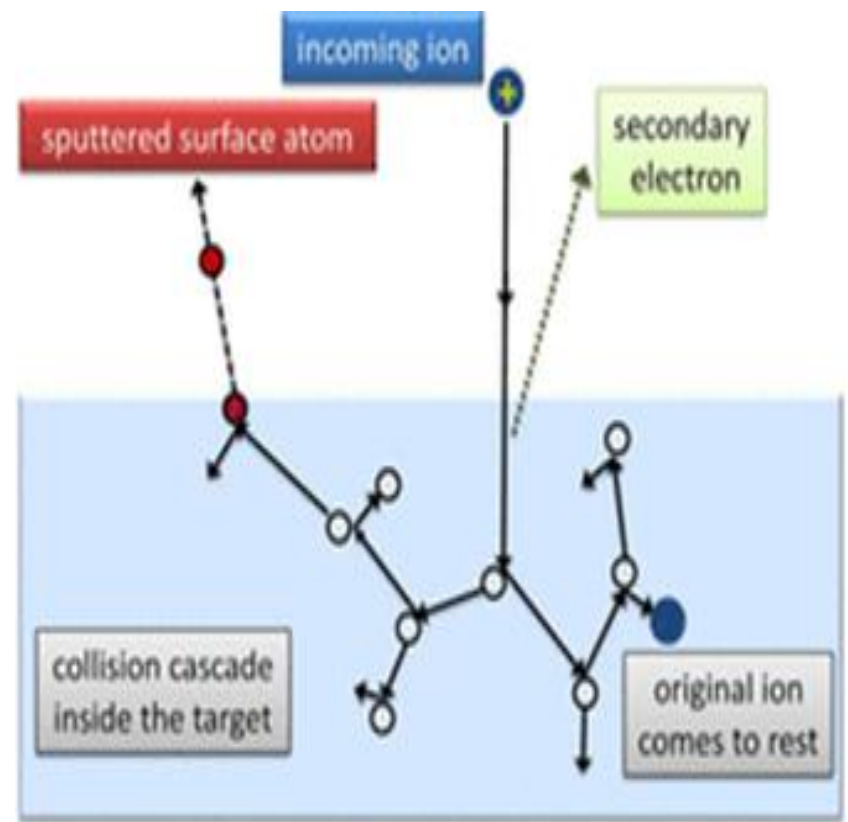

Fig.4. Schematic illustration of the sputtering process: inside the target, a cascade of collisions is triggered by an energetic ion knocking out one or more surface atoms.

The propagation of the cascade of collisions, the depth of ion penetration, depends on the composition and structure of the target, on the mass and energy of ions, on the intensity of the processes as a whole [9].

Thus, the synthesis of conductive oxide and dielectric coatings by the PMSD method with a rotating magnetic block is extremely universal. The coating formation mechanisms implemented in it, new physicochemical mechanisms of the interaction of ionized gases with a metal target determine the more subtle effects of sputtering processes. The rotating magnetic system under the circular target cathode, which is sprayed, along its surface gives synchronous motion of a closed magnetic field. It is also important that technological parameters can be adjusted, such as the distance between the permanent magnets of the opposite poles and the configuration of their location. This makes it possible to change the configuration of the magnetic field on the target surface and, accordingly, the intensity distribution of the ion bombardment on it. Another parameter is the speed of rotation of the magnetic system[10, 11]. As a result, high uniformity of the composition of thin dielectric and transparent conductive films is ensured. Interestingly, in the scanning mode, because it is possible to more effectively control the flow of ion bombardment of the target, which leads to a decrease or disappearance of the hysteretic behavior of the deposition process, the elimination of "sparking" and "disappearance of the anode" and an increase in the deposition rate. The energy distribution of individual types of ions bombarding the target and the composition of the total flow of molecules on the substrate are key to characterizing reactive magnetron sputtering and to a good understanding of the deposition processes.

\section{INTENDED AND PROMISING APPLICATIONS OF OUR MAGNETRON}

Modern materials and technologies are gaining special importance today, because the challenges we face today are unimaginable without the development of modern technologies needed to obtain thin tapes.

It is important for us to use this technology to get transparent and conductive oxide and absorption layers, which must be used in solar cells in combination with layers of perovskite. This example of use is of great importance, as only low-temperature coatings can be used with perovskite. And modern solar energy converters are unimaginable without perovskite.

\section{CONCLUSION}

Today, the world is facing global challenges, and environmental issues are the most important. That is why modern technology is of great importance. From them you can get materials that can solve important issues for the future of mankind. The most important of these problems is the search for alternative energy sources. Using solar energy as an alternative source of energy is what successful scientists are working on. It is in the context of work on these issues that it will be possible to discuss the proposed innovative technology and, in particular, methods for obtaining the necessary films for modern solar cells.

\section{ACKNOWLEDGMENTS}

The author very gratefully acknowledges the critical reading of entire early drafts of this article and the many useful suggestions of several colegae and excellent scientists:

PhD Zaur Berishvili, senior scientist LEPL Research Institute "Optica”, ass. professor Caucasus University;

PhD Irakli kordzaxia, chief scientist, head of department LEPL Research Institute "Optica”;

PhD Guram Dekanozishvili, senior scientist Andronikashvili Institute of Physics.

The author also thanks other scientists and authors whose works were used during the writting of this article.

Note: The use of patent materials in the article is agreed with the author of the patents, $\mathrm{PhD}$ Zaur Berishvili (see reference)

\section{REFERENCES}

[1] Corbani, J.F. (1975) "Cathode Sputtering Apparatus". Patent USA 3 878085 Priority 5/07/1973, Date of publication 15/04/1975.

[2] Chapin, J.S. (1979) "Sputtering Process and Apparatus". Patent USA 4166 018, Priority 31/01/1974, Date of publication 28/08/1979.

[3] Chapin, J.S. (1974) „Planar Magnetron” Journal of Vacuum Science \& Technology Research Development 25 (1), pp. 37-40.

[4] Berishvili, Z., Skhiladze, G., and Shioshvili, S. (1983), "The Arrangement of Ion-Plasma Sputtering in Vacuum". Author's Certificate, № 1160761 (USSR), Date of publication 8/02/1985.

[5] Berishvili, Z., Gadakhabadze, I., Skhiladze, G., and Shioshvili, S. (1986), "The Arrangement of Ion-Plasma Sputtering of Materials in Vacuum”. Author's Certificate, № 1244960 (USSR), Date of publication 15/03/1986.

[6] Berishvili Z., Gadakhabadze I., Kordzakhia I., and Dekanozishvili G. (2017), "Planar Magnetron Sputtering Device: A New Generation of Magnetron Sputtering Design and Technology". Journal of Physical Science and Application, vol. 7, no. 2, pp. 28-39. 
[7] Bräuer, G. (2014), Comprehensive Materials Processing, Films and Coatings: Technology and Recent Development, Reactive Sputtering, Elsevier.

[8] Breg,S. Blom, H-O, Larsson,T. and Nender,C. (1987), „Modeling of reactive sputtering of compound materials". Journal of Vacuum Science \& Technology A 5, pp. 202-207.

[9] Depla D, De Gryse R. (2004), "Target poisoning during reactive magnetron sputtering" Part I: the influence of ion implantation, Surface and Coatings Technology, Volume 183, Issues 2-3, pp.184-189

[10] Berishvili, Z. (2016), "Planar Magnetron Sputtering Device". GE P 2016 6512 B, 28.05.2015, Date of publication, 2016.03.25. № 6, International Application of PCT/EG2016/000005, № WIPO/WO 2016/189337, Date of publication, 01 December 2016.

[11] Berishvili, Z. (2019), "Method for Production of Nanomaterial in Vacuum and Magnetron Sputtering Device for its Embodiment", EG P 20197026 B, 22.05.2018, Date of publication, 2019.05.27, № 10, International Application PCT/GE2019/050002, № WIPO/WO 2019/224564, Publication Date 28.11.2019.

\section{Author's short biographical note}

Ms. Marina Kipiani was awarded the qualification of metallurgical engineer in physical metallurgy, equipment and technology of metals heat treatment. In 2017 she was awarded PhD degree in materials science. Ms. Marina Kipiani currently works at the LEPL Research Institute "Optica" as a senior scientist and holds the position of deputy head of the department of optical research. At this stage, she is working on innovative technologies for receiving advanced materials under the guidance of senior scientist LEPL Research Institute "Optica", Ass. Professor Caucasus University, PhD Zaur Berishvili.

She is a participant of various scientific grant competitions, a participant in the application for the grant competition announced by the ERC and one of the winners of the 2019 Shota Rustaveli National Scientific Foundation of Georgia. 